\title{
Participación ciudadana en la gestión bibliotecaria: reflexiones teóricas y aplicación práctica
}

Citizen participation in library management: theoretical reflections and practical application

\author{
João de Sousa GuerReiro, Ana Belén Ríos HiLARIo \\ Facultad de Traducción y Documentación, Universidad de Salamanca, España, \\ jsousaguerreiro@gmail.com, anarihi@usal.es
}

\begin{abstract}
Resumen
La participación ciudadana es un concepto cada vez más presente en la literatura sobre gestión de los servicios públicos, en general, y de las bibliotecas en particular. Dicho concepto se refiere a la inclusión gradual de los ciudadanos en la toma de decisiones de los servicios. El presente trabajo pretende demostrar que la participación ciudadana puede ser entendida como un elemento básico de la gestión de bibliotecas, al permitir conocer lo que desea la ciudadanía y gestionar de forma más eficiente y eficaz los servicios bibliotecarios. Para ello, en primer lugar, se definen las características de la participación ciudadana y sus ventajas. En según lugar, se expone la experiencia realizada en la Biblioteca Pública de Salamanca, que confirma la aplicabilidad de las dinámicas participativas para establecer una relación normalizada con la comunidad y promover una ciudadanía activa. Concluye el artículo reflexionando sobre la aplicación de este movimiento en el entorno bibliotecario.
\end{abstract}

Palabras clave: Participación ciudadana. Gestión de servicios públicos. Gestión bibliotecaria. Biblioteca Pública de Salamanca. Salamanca (España).

\section{Introducción}

En los últimos años los servicios públicos son un tema recurrente en el debate político y social. Los recortes presupuestarios, los casos de corrupción y mala gestión o privatización afectan a la imagen del sector público. Debido a esto, la ciudadanía demanda "que las actividades de la administración sean lícitas, legales, pero además reclaman cada vez más que sean efectivas y eficientes" (Lozano Díaz, 2006, p. 43). En la misma línea Obregón \& Vilalta (citado por Lozano Díaz, 2006, p. 45) afirma que los ciudadanos "reclaman que cada vez más el cumplimento de la legalidad vaya asociado a una participación social proactiva de su administración, a un servicio más transparente y cercano a ellos".

Así se identifican tres ejes principales (Figura 1), que deben ser entendidos como facetas interdependientes de una misma realidad, en la transformación del concepto de servicio público.

\begin{abstract}
Citizen participation is a concept that is increasingly present in the literature on the management of public services in general and of libraries in particular. This concept refers to the gradual inclusion of citizens in the decision making of services. This paper aims to demonstrate that citizen participation can be understood as a basic element of library management, allowing to know what citizens want and to manage library services more efficiently and effectively. For that, first the characteristics of citizen participation and its advantages are defined. Secondly, indicated the experience at the Salamanca Public Library is presented, which confirms the applicability of participatory dynamics to establish a normalized relationship with the community and promote active citizenship. The article concludes by reflecting on the application of this movement in the librarian environment.
\end{abstract}

Keywords: Citizen participation. Public management. Library management. Public Library of Salamanca. Salamanca (Spain).

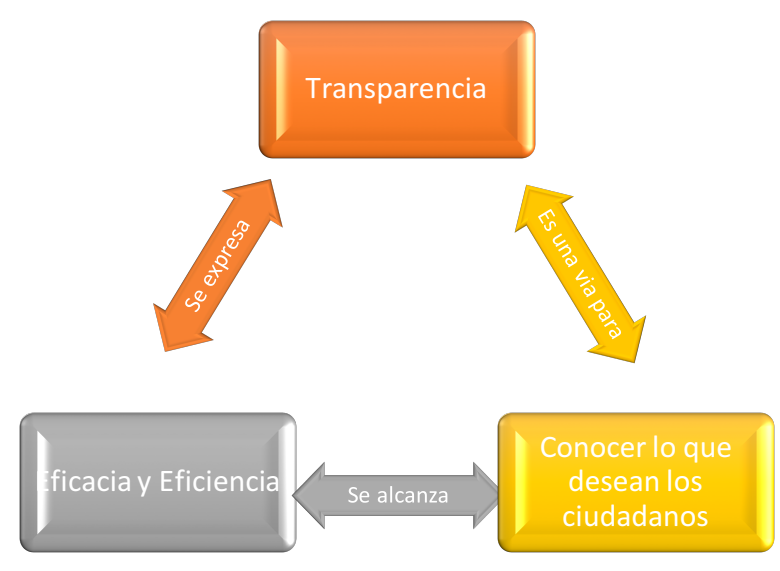

Figura 1. Ejes de la transformación de los servicios públicos

La eficiencia y eficacia son conceptos que provienen del ámbito del marketing y tienen una relación directa con la planificación estratégica. No se trata solamente de alcanzar una meta o 
los objetivos de la institución (eficacia), sino de escoger el mejor camino para alcanzarla (eficiencia). Se entiende por eficiencia, el correcto uso de los recursos humanos y materiales, siempre teniendo en cuenta los intereses del público. El término eficiencia está relacionado con la creación de una metodología que permita en un futuro reproducir los logros alcanzados y, para ello, es necesario documentar las acciones de gestión de las instituciones. Eficacia y eficiencia están asociados a la imagen que la comunidad tiene de la institución. Para crear una imagen positiva de la organización, es decir, que se considere como una institución eficaz y eficiente, es necesario que los usuarios tengan acceso a los datos sobre los servicios para así poder evaluarlos. Es en este punto en el que entra en juego el concepto de transparencia.

Dicho concepto, está directamente asociado a la producción de documentos que expresen el trabajo y la gestión de los servicios los cuales deben ser accesibles a los ciudadanos. Con la creación de estos documentos se busca no solo comunicar la legalidad de los servicios, sino demostrar que éstos son justos, es decir, que utilizan los recursos públicos de la mejor forma posible y que tienen en cuenta la comunidad a la que sirven. Según recoge la Ley de Transparencia Española (2013, p. 97924)

[...] sólo cuando la acción de los responsables públicos se somete a escrutinio, cuando los ciudadanos pueden conocer cómo se toman las decisiones que les afectan, cómo se manejan los fondos públicos o bajo qué criterios actúan nuestras instituciones podremos hablar del inicio de un proceso en el que los poderes públicos comienzan a responder a una sociedad que es crítica, exigente y que demanda participación de los poderes públicos.

La tensión dialéctica, que existe entre la función que tiene o debe tener un servicio público como una biblioteca y las nuevas corrientes de la gestión, que abogan por que una institución sea solamente lo que sus usuarios o los ciudadanos demanden de ella, es una cuestión difícil de resolver. La mayor parte de las veces defender categóricamente cualquiera de las dos posiciones lleva a un callejón sin salida. Por un lado, la biblioteca tiene un papel social que le viene dado por su recorrido histórico como institución pública de cultura y educación. Por otro lado, es importante tener en cuenta que la biblioteca se inserta en una sociedad cambiante y que el público es el valor máximo de cualquier institución, sea pública o privada. Es necesario encontrar un término medio que le permita cumplir las funciones para las que fue creado el servicio y, al mismo tiempo, se pueda adaptar a las nuevas necesidades de la comunidad. En el caso de las bibliotecas públicas se puede pensar en una institución que promueva la cultura y sea un espacio de acceso igualitario a la información, siempre teniendo en cuenta la opinión y necesidades de los ciudadanos. No se trata de transformar todo lo que fue creado hasta el momento, sino de saber qué servicios o actividades potenciar, cuáles se pueden mejorar o qué nuevas demandas necesitan respuesta. Por ello, resulta inevitable contar con la opinión de los ciudadanos y conocer sus necesidades y expectativas sobre el organismo. En muchos casos no se trata de saber qué función o servicios deben tener instituciones como la biblioteca, sino de conocer la mejor forma de prestar dichos servicios a su comunidad.

Muchos son los métodos y técnicas para conocer las necesidades de la ciudadanía, desde las menos directas, como las encuestas, hasta las que se relacionan más íntimamente con los ciudadanos, como los grupos de discusión y demás técnicas participativas.

Partiendo de las anteriores premisas, el presente trabajo pretende demostrar que la participación ciudadana puede ser entendida como un elemento básico de la gestión de bibliotecas, para conocer lo que desea la ciudadanía y para gestionar de forma más eficiente y eficaz los servicios bibliotecarios. Para ello, en primer lugar, se definirán las características de la participación ciudadana y sus ventajas. En según lugar, se mostrará la experiencia realizada en la Biblioteca Pública de Salamanca, que viene a probar la aplicabilidad de las dinámicas participativas para establecer una relación normalizada con la comunidad y promover una ciudadanía activa.

\section{Participación ciudadana: una respuesta posible}

En este contexto de cambios institucionales y de nuevas demandas de los servicios públicos por parte de los ciudadanos, la participación ciudadana se alza como una respuesta con bastante aceptación por parte de la ciudadanía en general, debido principalmente a los movimientos de la sociedad civil que surgieron después de la crisis económica internacional de 2008.

\subsection{Definición del concepto}

La participación ciudadana es un término de difícil definición, al no haber un consenso generalizado entre los estudiosos, pero existen algunos puntos convergentes (Figura 2) que posibilitan acercarse a este concepto. 


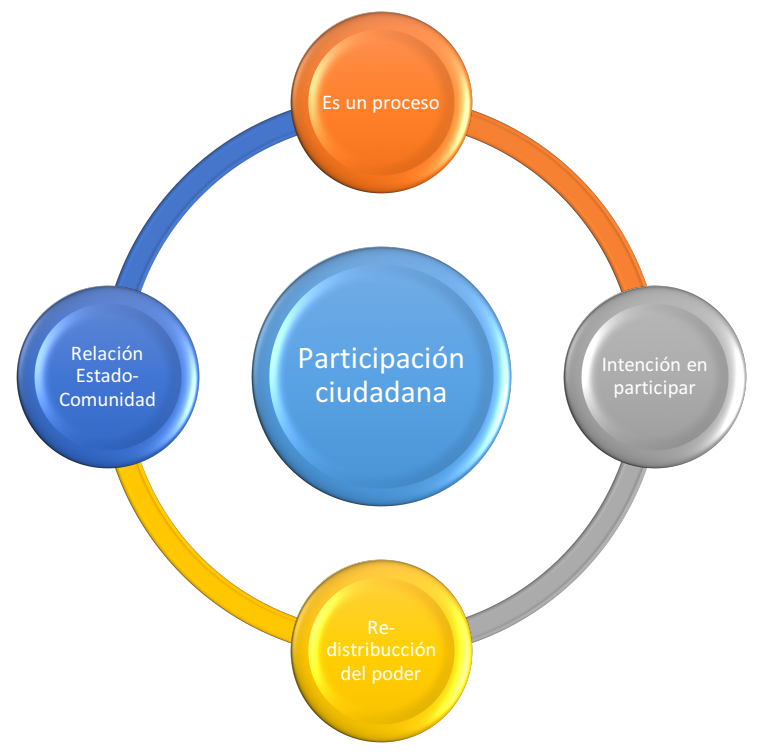

Figura 2. Principales características de la participación ciudadana

De forma resumida se puede entender la participación ciudadana como "todas aquellas prácticas políticas y sociales a través de las cuales la ciudadanía pretende incidir sobre alguna dimensión de aquello que es público" (Parés, 2009, p. 17). En esta primera aproximación se puede entrever la primera característica de este concepto: relación concreta entre la ciudadanía y los servicios del estado, donde se reflexiona y construye la idea de lo público (Álvarez, 1997; Ziccardi, 1998; Cunill Grau, 2008, Rodríguez Ponce y Pedraja Rejas, 2009; Oraisón, 2011).

La participación, según Espinosa, es una "acción emprendida deliberadamente por un individuo o conjunto de éstos" (2008, p. 74). La intencionalidad en participar es una característica destacada por diferentes autores, que se da tanto en el ámbito local como en un ámbito más general (Alberich Nistral, 2012), para formar parte en la toma de decisiones, integrarse en la comunidad o solucionar un problema o cuestión específica (Velásquez y González, 2003; Montesino, 2009).

El concepto de participación ciudadana suele también venir asociado a la inclusión de los ciudadanos en la gestión de los servicios públicos en general y en la toma de decisiones en particular. Bajo este hecho subyace la redistribución de poder (Arnistein, 1969; Cunil Grau, 2008; Merino, 2013), es decir, que los ciudadanos conozcan de forma directa las especificidades del servicio y puedan actuar sobre el mismo ya sea mediante la expresión de su opinión, en la determinación de los objetivos o colaborando y votando en las reuniones de creación de los planos estratégicos. Consecuentemente, esta integración de los ciudadanos en las decisiones permite a la institución conocer lo que desean los ciudadanos, promueve una mejora en la imagen institucional como servicios abiertos al ciudadano, al mismo tiempo que fortalece una ciudadanía activa y un sentido de pertenencia comunitaria.

La última característica que define la participación ciudadana, y la única que reúne el consenso de los investigadores, es que es un proceso. Es decir, no se trata de un conjunto de actividades aisladas con un objetivo específico, por lo contrario, debe ser entendida como un proyecto a largo plazo en el cual tanto la ciudadanía como las instituciones intentan establecer espacios de reflexión y debate público que propicien el aprendizaje y la creación de una conducta responsable, orientada al establecimiento de estrategias creativas para satisfacción de los intereses mutuos o encontrar las soluciones más favorables para todos (Halal, 2001; Antonacopoulou y Meric, 2005; Giraldo et al., 2010).

Combinando las diferentes características anteriormente descritas se puede elaborar una posible definición de participación ciudadana como el proceso que a través de la utilización de diversos métodos y técnicas promueve la creación de una relación bilateral e intencionada entre el poder institucional o el Estado y la ciudadanía, con el objetivo de permitir una redistribución más equitativa del poder en la gestión de los servicios públicos.

\subsection{Ventajas de la participación ciudadana}

Las ventajas de la inclusión de los ciudadanos están directamente relacionadas con las características que se enunciaron en el apartado anterior. Las dinámicas participativas, entendidas como un conjunto de métodos y técnicas que permiten a los ciudadanos participar gradualmente en la toma de decisiones de los servicios públicos, permiten que las comunidades entiendan las instituciones del Estado como suyas y, consecuentemente, al activar el sentido de pertenencia, mejorar la reputación de las mismas. Al mismo tiempo, y debido a que esta relación es bilateral, permite a las instituciones conocer de forma directa la realidad social de la que son parte, diagnosticando con antelación los problemas que pueden surgir en el entorno y promoviendo servicios más adecuados a las necesidades de la comunidad.

La redistribución del poder de decisión, permite encontrar soluciones creativas y más adecuadas a las necesidades de la comunidad ya que resultan del trabajo en conjunto con la misma. 
Con la creación de espacios de reflexión sobre los servicios públicos los ciudadanos pueden entender de forma directa las limitaciones, por ejemplo, económicas y legales a las que se enfrentan las instituciones estatales y comprender que éstas deben optar por aquellas soluciones que son más beneficiosas para la ciudadanía en general. Al mismo tiempo, esa toma de conciencia, tanto de los servicios como de la comunidad, promueve el cambio de mentalidades; ya que, al incentivar el dialogo y la reflexión sobre la estructura social, permite que se abandonen los intereses individuales para adoptar una perspectiva más global o comunitaria.

La intencionalidad de la relación promueve que los diferentes grupos sociales y las instituciones tomen conciencia de que la participación depende también de ellos y puede ser un motor para una ciudadanía más activa. Normalmente se inicia la participación con los grupos más proactivos de la comunidad y es natural que otros grupos sigan la labor de los demás.

Al ser un proceso, permite que paulatinamente tanto las instituciones cómo los ciudadanos puedan aprender gradualmente como mejorar la relación y conseguir un trabajo conjunto más efectivo, lo que debe traducirse finalmente por la creación de una sociedad más democrática. Además debe comprenderse que la estructura social es compleja y que se componen de opiniones diferentes, incluso opuestas, y que lo que se espera es que en conjunto se tomen las decisiones más favorables para la sociedad en general.

\section{3. ¿Cómo llevar la participación ciudadana a la práctica?}

La participación ciudadana no es algo nuevo ni en la gestión de los servicios públicos en general - cuyo caso más significativo son los presupuestos participativos de Porto Alegre en Brasil (2016) - ni en el ámbito específico del sector bibliotecario, principalmente en los países anglosajones (Pacios Lozano, 2003). Se podrían enunciar diversos ejemplos de estos países, pero se ha optado por presentar una experiencia que desarrollaron los autores del presente artículo en la Biblioteca Pública de Salamanca, por tratarse de un ámbito más cercano geográfica y culturalmente. A pesar de tratarse de una institución con una gran relación con su comunidad no tenía una estrategia de participación ciudadana, de tal modo que se propuso dar los primeros pasos en la activación de una relación normalizada. Dicha experiencia figura claramente detallada en la tesis doctoral "Espacio compartido: modelo de relación biblioteca-comuni- dad basado en la participación ciudadana y la teoría de los stakeholders" (Guerreiro, 2017).

\section{1. ¿Con quién nos relacionarnos?}

Existen varios métodos y técnicas participativas, pero los centros no disponen de recursos, principalmente humanos y de tiempo, para establecer un contacto directo con todos los grupos de la comunidad. Así surge la cuestión de la representatividad de las dinámicas participativas, tema ampliamente debatido por los estudiosos de la materia (Noth, 1995; Gomá y Blanco, 2003; Cunill Grau, 2008; Casas, 2009). Teniendo en cuenta la intencionalidad de la relación, se indica que una "posibilidad es favorecer la participación de una red abierta de actores interesados y dispuestos a participar en los temas a tratar, ya sea por su implicación directa en ellos, ya sea por tener un interés genérico" (Brugué y Gallego citado por Gallego, Gomà y Subirats, 2003). Igualmente autores como Font, Blanco, Gomá y Jarque (2000, p. 125) afirman "la voz ciudadana que debe ser escuchada es la de los ciudadanos o los grupos que manifiestan un interés en expresarla, y no debe preocuparnos que no todos los ciudadanos estén representados en el proceso participativo". Además es necesario encontrar una forma que posibilite activar la participación y superar los obstáculos asociadas a estas dinámicas.

Asimismo, se debe tener en cuenta que además de las aportaciones teóricas las bibliotecas necesitan estrategias concretas que les permitan iniciar las dinámicas participativas. Así en la Biblioteca Pública de Salamanca se ha decidido trabajar con los grupos que tenían más relación con la institución, siguiendo la teoría de los stakholders. Dicha teoría fue creada por Edward Freeman (1984) en el ámbito de la gestión empresarial y tiene como principal objetivo promover que las decisiones tomadas sean las más beneficiosas para todos los grupos de interés que se relacionen con la organización. Dicha teoría guarda una estrecha relación con los principios de la participación ciudadana.

\subsection{Selección de los grupos}

Para determinar con que grupos o personas la biblioteca podría empezar sus dinámicas participativas se realizaron dos reuniones con los trabajadores de las diferentes secciones de la biblioteca y de diferentes categorías profesionales.

En la primera reunión se hizo una tormenta de ideas con el objetivo de identificar todos los grupos que se vinculaban con la biblioteca. En esa reunión se identificaron 74 grupos sociales con quien la biblioteca se relacionaba que pos- 
teriormente fueron categorizados en las siguientes 10 categorías (figura 3 ).

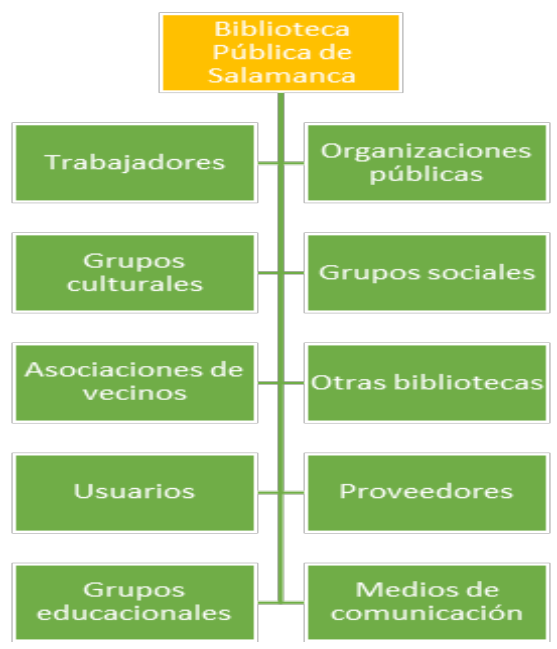

Figura 3. Categorías de stakeholders de la Biblioteca Pública de Salamanca

En la segunda reunión se determinaron los grupos con los que se iniciaría el proceso de relación. Dicho proceso se realizó mediante la reflexión de los trabajadores sobre el nivel de relación y ubicando las 10 categorías identificadas previamente en un cuadro de priorización (Tabla I) según dos criterios: a) influencia: aquellos que tienen mayor impacto en el logro de los objetivos de la biblioteca o mayor importancia en la concretización del objeto de estudio; y b) dependencia: aquellos que se ven más afectados positiva o negativamente por la actividad de la biblioteca.

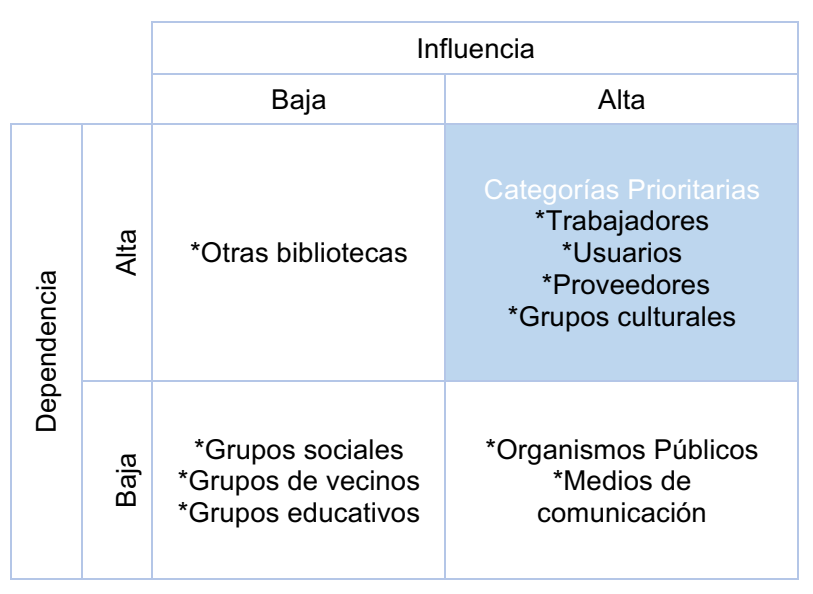

Tabla I. Priorización de las categorías

Así, se determinaron que las categorías prioritarias, aquellas que son más influyentes e influenciadas en la toma de decisiones de la biblioteca, son: usuarios, proveedores, grupos culturales y los propios trabajadores.

\subsection{Contactar con las categorías prioritarias}

Posteriormente se convocaron a los representantes de los diferentes grupos de cada una de las cuatro categorías prioritarias. Se realizaron 4 reuniones, una por cada categoría. Como el propósito era activar la participación ciudadana se decidió establecer un tema, que todos participantes pudieran conocer, de este modo se hizo una evaluación del trabajo diario de la biblioteca centrado en cuatro aspectos: colección (calidad, cantidad y organización), personal, espacio y horarios, y actividades. Cada reunión tenía un moderador y un secretario que guiaban la misma, proponiendo un conjunto de cuestiones creadas a priori. Las reuniones se desarrollaron como una conversación informal sobre el trabajo de la biblioteca y siguieron la estructura y los principios de los grupos de discusión. Se optó por una técnica grupal, pues permite mayor espontaneidad de los participantes, además de promover el debate y la reflexión en conjunto (Llopis Goig, 2004). La información de las reuniones fue registrada por el moderador y el secretario, además de ser grabada en video para la posterior visualización y confirmación de datos. En este contexto la técnica de grupos de discusión no posee unas perspectiva sociológi$\mathrm{ca}$, intentando averiguar las razones que subyacen a la opinión de los participantes, por el contrario, en las dinámicas participativas se registra todo aquello que los participantes expresan pudiendo establecer una breves notas a modo de observaciones o contexto (Tabla II).

\begin{tabular}{|c|c|c|}
\hline & Información objetiva & Observaciones \\
\hline \multirow[t]{2}{*}{$\begin{array}{l}0 \\
0 \\
0 \\
\frac{\pi}{0} \\
.0 \\
.0 \\
0 \\
\end{array}$} & $\begin{array}{l}\text { ¿Qué conocen? } \\
\text { Debates, ciclos de cinema, } \\
\text { clubes de lectura, talleres de } \\
\text { conversación, encuentros con } \\
\text { autores, visitas escolares }\end{array}$ & $\begin{array}{l}\text { Los más jóvenes no } \\
\text { conocían los clubes } \\
\text { de lectura. }\end{array}$ \\
\hline & $\begin{array}{l}\text { Las actividades son variadas y } \\
\text { muy bien valoradas }\end{array}$ & \\
\hline
\end{tabular}

Tabla II. Resultados del grupo de usuarios

Para el análisis de la información el moderador y el secretario tabularon la información siguiendo el orden de las cuestiones presentadas en las reuniones, a través de la información registrada durante la sesión y la repetida visualización de los registros audiovisuales. Al no recurrir a la transcripción de las reuniones todo el proceso se hace más rápido, entendiendo que aunque la transcripción sea habitual en las técnicas grupales ésta "no es en sí misma el soporte privilegiado sobre el cual hacer el análisis de los grupos" (Gutiérrez Brito, 2008, p. 134), pues toda transcripción es un proceso interpretativo 
no más objetivo que los demás documentos secundarios.

\subsection{Resultados}

Muchas fueron las conclusiones de la aplicación de las dinámicas participativas en la Biblioteca Pública de Salamanca, como se indican en la tabla III. Posteriormente algunas de ellas fueron agrupadas en dos objetivos estratégicos: a) mejorar la difusión y la comunicación externa e interna; y b) Mejorar la dinámica de trabajo para facilitar la creación de una conciencia de equipo.

Los propios trabajadores decidieron que se debería trabajar este último punto, pues en las diferentes fases de la dinámica grupal —desde las reuniones para identificar los grupos que se relacionaban con la biblioteca hasta la última reunión para debatir los resultados- se dieron cuenta que no conocían el trabajo desempeñado por sus compañeros $y$, consecuentemente, no conocían la labor total de la institución ni su verdadera importancia social. El personal señaló que esa nueva conciencia los motivaba a seguir trabajando y decidieron divulgar las actividades de la biblioteca en sus zonas de residencias y a través de sus cuentas personales en las diferentes redes sociales.

Asimismo, los otros participantes consideraron que era un honor ayudar a la biblioteca a mejorar sus servicios, y creen que en un futuro todos los servicios públicos tendrán en cuenta la participación ciudadana en su gestión. Se debe señalar que ninguno de los participantes había colaborado en una dinámica participativa anteriormente.

\begin{tabular}{|c|c|}
\hline Resultado & Comentario \\
\hline Opinión general & $\begin{array}{l}\text { Imagen positiva de la biblioteca como un centro activo e innovador. La biblioteca se considera un } \\
\text { espacio de encuentro y acceso a la cultura. Esta imagen guarda una estrecha relación con la } \\
\text { programación de actividades de la biblioteca. }\end{array}$ \\
\hline Personal & Es amable y siempre intenta solucionar los problemas que se le presentan \\
\hline Actividades & $\begin{array}{l}\text { La biblioteca programa muchas actividades y bastante variadas. Las actividades confieren a la } \\
\text { institución una imagen innovadora (pionera en muchos casos) y a contracorriente (muchas } \\
\text { instituciones han suspendido la programación de actividades debido a los recortes, pero la biblioteca } \\
\text { ha seguido programando con presupuestos reducidos). }\end{array}$ \\
\hline Falta de divulgación & $\begin{array}{l}\text { Muchas veces las personas solo conocen las actividades, después de que estas sucedan. Hay que } \\
\text { mejorar la divulgación a través de las diversas vías de comunicación. Los documentos divulgativos no } \\
\text { poseen una identidad propia, que permita reconocer que son de la biblioteca. }\end{array}$ \\
\hline Sección infantil & No comprenden porque la biblioteca no tiene una colección para niños. \\
\hline $\begin{array}{l}\text { Organización de la } \\
\text { colección }\end{array}$ & $\begin{array}{l}\text { La disposición de la colección y su organización es complicada y a veces poco intuitiva, } \\
\text { principalmente en los centros de interés. Falta una señalización más clara. }\end{array}$ \\
\hline $\begin{array}{l}\text { Falta de poder de } \\
\text { decisión o autonomía de } \\
\text { los trabajadores }\end{array}$ & Crea una imagen controladora del cuadro de mando de la biblioteca. Ralentiza el trabajo. \\
\hline $\begin{array}{l}\text { Falta de organización del } \\
\text { trabajo }\end{array}$ & $\begin{array}{l}\text { Este punto está directamente relacionado con el anterior. Crea la sensación de que los trabajadores } \\
\text { no conocen totalmente su trabajo. Siempre necesitan confirmar lo que deben hacer. Ralentiza el } \\
\text { trabajo. }\end{array}$ \\
\hline Equipamiento malo & Ordenadores, fotocopiadoras e internet funcionan mal. Ralentiza el trabajo. \\
\hline $\begin{array}{l}\text { Poco cuidado con las } \\
\text { instalaciones }\end{array}$ & $\begin{array}{l}\text { Falta de limpieza en general, desorden de los materiales y cierto descuido. } \\
\text { Hay que mantener los espacios más limpios y ordenados. }\end{array}$ \\
\hline Falta público joven & Existe la idea de que a las actividades de la biblioteca no asiste el público joven. \\
\hline
\end{tabular}

Tabla III. Principales resultados obtenidos

\section{Conclusiones}

La participación ciudadana es una realidad consolidada en muchos países, principalmente anglosajones, y en el norte de Europa, y es solo una cuestión de tiempo que lo sea también en España. Los primeros pasos ya fueron dados, el termino ya se incorporó al lenguaje cotidiano, por los nuevos partidos que surgieron después de la crisis de 2008 y por muchas asociaciones y colectivos sociales. Además se debe entender que las nuevas tecnologías, promueven el ideal de participación.
Las demandas de una nueva gestión pública ya están ahí, cabe ahora a los cuadros de mando y a los trabajadores de dichas instituciones abrirlas a la participación de los ciudadanos. Igualmente la aplicación de dinámicas participativas en el sector bibliotecario vienen a ampliar una función que siempre fue suya, la de ser un pilar en la creación de una ciudadanía fuerte, no solo permitiendo el acceso igualitario a la información, sino promoviendo una práctica verdaderamente democrática. 
Además desde el ámbito bibliotecario ya se está reflexionando sobre la importancia de las bibliotecas como centros de creación de comunidad, tanto a nivel internacional -Declaración de Lyon sobre el acceso a la información y el desarrollo (IFLA, 2014) - o a nivel nacional -el informe de Prospectivas 2020 (CCB, 2013)-, que en el punto 6, asegura que una de las posibles líneas de desarrollo bibliotecario es que estas instituciones se conviertan en el Ágora de sus comunidades.

Para que esta afirmación se convierta en realidad no solo es necesario trabajar en favor de la sociedad a la que sirven, sino establecer métodos concretos para abrir las instituciones a la ciudadanía, permitiendo alcanzar el tan deseado objetivo de crear una biblioteca para y con la comunidad. Y como afirma la Nobel de Medicina y pensadora Rita Levi-Montalcini, "la responsabilidad es de quienes, en cada ámbito de la vida social, tienen ante sí los botones de mando" (2012, p. 98).

\section{Referencias}

Alberich Nistal, T. (2012). Guía fácil de la participación ciudadana: manual de gestión para el fomento de la participación en ayuntamientos y asociaciones. $2^{\mathrm{a}}$ ed. Madrid: Dykinson. ISBN: 978-84-9031-120-2.

Álvarez E., L. (coordinadora) (1997). Participación y democracia en la ciudad de México. México: Universidad Nacional Autónoma de México, 1997

Antonacopoulou, E.; Meric, J. (2005). From power to knowledge relationships: Stakeholder interactions as learningpartnerships. // Bonnafous-Boucher, M. \& Pesqueux,, Y (Ed.). Stakeholder Theory: an European prespective. London: Palgrave Macmillan, 2005. 125147.

Arnstein, S. R. (1969). A ladder of citizen participation. // Journal of the American Institute of Planners. 35:4 (Julio 1969) 216-224. http://rua.ua.es/dspace/bitstream/10045/1 6699/2/ESCALERA_ARNSTEIN_1969.pdf (21-03-2017).

Guerreiro, J. S. (2017). Espacio compartido: modelo de relación biblioteca-comunidad basado en la participación ciudadana y la teoría de los stakeholders (tesis doctoral). Dirigida por Ana Ríos. Salamanca: Universidad de Salamanca, 2017.

Brasil. Perfeitura Municipal de Porto Alegre (2016). Orçamentos participativos.h ttp://www2.portoalegre.rs.gov.br/ op/default.php?p_secao=1 (27-03-2017)

Casas, E. (2009). Representación política y participaciónciudadana en las democracias. // Revista mexicana de ciencias políticas y sociales. 15:205 (ene./abril. 2009) 59-76. http://www.scielo.org.mx/pdf/rmcps/v51n205/v51 n205a4.pdf

CCB: Consejo de Cooperación Bibliotecaria (2013). Prospectiva 2020 Las diez áreas que más van a cambiar en nuestras bibliotecas en los próximos años. Madrid: CCB, 2013. http://www.ccbiblio.es/wp-content/uploads/Estudio _prospectiva_2020.pdf. (20-03-2017).

Cunill Grau, N. (2008). La construcción de ciudadanía desde una institución pública. // Mariani, R. (coord.) Contribuiciones al debate, vol.2: Democracia, estado, ciudadanía: hacía un Estado de y para la democracia en América Latina. Lima: Programa de la Naciones Unidas para el
Desarrollo, 2008. ISBN: 978-9972-612-30-5 113-138. http://www.iidh.ed.cr/multic/UserFiles/Biblioteca/IIDHSeg uridad/12_2010/be846c2a-a0e6-44d0-9fae-5d9d637df 9ff.pdf (21-03-2017).

España. Ley 19/2013, de 9 de diciembre, de transparencia, acceso a la información pública y buen gobierno. // BOE 295 (10 de diciembre de 2013). https://www.boe.es/ boe/dias/2013/12/10/pdfs/BOE-A-2013-12887.pdf (2703-2017)

Espinosa, M (2009). La participación ciudadana como una relación socio-estatal acotada por la concepción de democracia y ciudadanía. // Andamios. 5:10 (México abr. 2009), 71-109. Dossier: Ciudadanía y representación. ISSN 1870-0063. http://www.scielo.org.mx/scielo.php? script=sci_arttext\&pid=S1870-00632009000100004 (2403-2017)

Font, J.; Blanco, I.; Gomá, R.; Jarque, M. (2000). Mecanismos de participación ciudadana en la toma de decisiones locales: una visión panorámica. // XIV Concurso de Ensayos CLAD: Administración pública y ciudadanía. Caracas, Venezuela:CLAD, 2000

Freeman, R. E. (1984). Strategic management: a stakeholder approach. Boston: Pitman, 1984. ISBN: 0273019139

Gallego, R.; Gomá, R.; Subirats, J. (2003). Estado de bienestar y Comunidades Autónomas. Madrid: Tecnos, 2003. ISBN: 84-309-3941-5.

Giraldo, N.; Hincapié, L.; Zapata, C. y Sánchez, L. (2010). Hacia la renovación de concepciones y prácticas de la planeación y el desarrollo. http://dintev.univalle.edu.co/ revistasunivalle/index.php/prospectiva/article/view/368

Gomá, R.; Blanco, I. (2003). Gobiernos locales y redes participativas: retos e innovaciones. // Revista CLAD: reforma y democracia, no. 26, (junio 2003), 1-15. http://siare.clad.org/revistas/0043507.pdf (20-03-2017)

Gutiérrez Brito, J. (2008). Dinámica del grupo de discusión. Madrid: Centro de investigación sociológica, 2008. ISBN: 978-84-7476-450-5.

Halal, W. E. (2001). The collobarative entreprise: a stakeholders model uniting profitability and responsibility. // Journal of corporate citizenship. 2 (summer 2001) 27-42. http://home.gwu.edu/ halal/Articles/Collaborative \%20Enterprise.pdf (19-03-2017)

IFLA (2014). Declaración de Lyon sobre el acceso a la información y el desarrollo. La Haya: IFLA, 2014. http://www.lyondeclaration.org/content/pages/lyondeclaration-es-v2.pdf (17-03-2017)

Levi-Montalcini, R. (2012). Tiempo de acción: el mundo global y el nuevo siglo. Barcelona: RBA, 2012. ISBN: 978-84-9006-361-4.

Lozano Díaz, R. (2006). La biblioteca pública del siglo XXI atendiendo clientes, movilizando personas. Gijón: Trea, 2006. ISBN: 84-9704-251-4.

Llopis Goig, R. (2004). Grupos de discusión. Madrid: ESIC, 2004. ISBN: 84-7356-373-5

Merino, M. (2013). La participación ciudadana en la democracia. México D. F.: Instituto Federal Electoral, 2013. ISBN: 968-658-68-5. http://www.ine.mx/archivos3/portal/ historico/recursos/IFE-v2/DECEYEC/DECEYECCuadernosDivulgacion/2015/cuad_4.pdf (19-03-2017)

Montesino, E. (2009). El presupuesto participativo en América Latina. ¿Complemento o subordinación a la democracia representativa? // Revista Reforma y Democracia. 44. http://siare.clad.org/fulltext/0062100.pdf

North, D. C. (1995). Instituciones, cambio institucional y desempeño económico. México: Fondo de Cultura Económica, 1995. ISBN: 968-16-3982-0.

Oraisón, M. (2016). La Participación como Generadora y Garante de Democracia y Ciudadanía. // Revista Internaci- 
onal de Educación para la Justicia Social. 5:1 (2016) 89107. https://revistas.uam.es/riejs/article/view/4345/4718.

Pacios Lozano, A. R. (2003). La participación de los ciudadanos en las propuestas de futuro de la biblioteca pública. // Boletín de la Asociación Andaluza de Bibliotecarios. 73 (diciembre 2003) 69-84. http://www.iesa.csic.es/ eventos/adjunto1_9OCTUBRE_2009.pdf. (22-03-2017)

Parés, M. (2009). Introducción: participación y evaluación de la participación. // Parés, M. (coord.). Participación y calidad democrática: evaluando las nuevas formas de democracia participativa. Barcelona: Ariel, 2009. 15-26. ISBN: 978-84-344-1839-4

Rodríguez Ponce, E.; Pedraja Rejas (2009). Análisis del impacto del proceso de toma de decisiones estratégicas sobre la eficacia de las organizaciones públicas // Revis- ta Innovar. 19:35. http://www.redalyc.org/pdf/818/818 19026004.pdf

Velásquez, C., F.; González R. E. (2003), ¿Qué ha pasado con la participación ciudadana en Colombia?. Bogotá: Fundación Corona, 2003.

Ziccardi, A. (1998), Gobernabilidad y Participación Ciudadana en la Ciudad Capital. México: Instituto de Investigaciones Sociales (Universidad Nacional Autónoma de México), 1998.

Enviado: 2017-03-31. Segunda versión: 2017-06-20. Aceptado: 2017-06-20 